cases ( $\mathrm{N}:$ 14). Mean time of hospitalization was 7.84 days (4.16 SD; max:15, min:1). Few patients had complications: surgical urologic complications: $12.0 \% \mathrm{~N}: 3$; postsurgery complications: urological $(\mathrm{N}: 2)$, abdominal wall infection $(\mathrm{N}: 2)$, eventration $(\mathrm{N}: 1)$, vascular $(\mathrm{N}: 1)$.

Conclusion* Ovarian cancer in premenopausal women is a threatening condition, diagnosed in most cases in advanced stages, that needs a combination of quemotherapy and surgery. Surgical approach must be aggressive in order to achieve a complete resection of the tumor.

\section{THE IMPACT OF ERAS IN CYTOREDUCTION FOR ADVANCED OVARIAN CANCER}

${ }^{1} \mathrm{D}$ Tsolakidis, 'D Zouzoulas*, 'E Markopoulou, ${ }^{1} \mathrm{~K}$ Chatzistamatiou, ${ }^{1} \mathrm{C}$ Zymperdikas, ${ }^{1} \mathrm{C}$ Anthoulakis, ${ }^{1} \mathrm{~T}$ Mikos, ${ }^{1} \mathrm{~L}$ Zepeiridis, ${ }^{1} \mathrm{E}$ Mpili, ${ }^{1} \mathrm{G}$ Pados, ${ }^{2} \mathrm{~A}$ Papanikolaou, ${ }^{1} \mathrm{G}$ Grimbizis. ${ }^{1}$ Aristotle University of Thessaloniki, 1st Department of Obstetrics and Gynecology, Thessaloniki, Greece; ${ }^{2}$ Aristotle University of Thessaloniki, 2nd Department of Obstetrics and Gynecology, Thessaloniki, Greece

\subsection{6/ijgc-2021-ESG0.413}

Introduction/Background* Complete cytoreduction is the cornerstone of the treatment for advanced ovarian cancer (AOC). To achieve this goal multiple organ resection is required, with an important impact on the patient's overall health. These patients may benefit from the implementation of enhanced recovery after surgery (ERAS). The aim of this study is to evaluate the possible benefit in the patient's postoperative morbidity.

Methodology Retrospective analysis of women with AOC from the $1^{\text {st }}$ Department of Obstetrics \& Gynecology AUTh at "Papageorgiou" Hospital (ESGO Certified Center for AOC), 2014 - 2019. From 2014 to 2016 conventional management (CM) was applied, while from 2017 to 2019 patients where managed with ERAS protocol. Patient \& tumor characteristics, treatment options and follow-up information were collected. Primary outcomes where ICU admittance, post-operative complications (Clavien - Dindo classification) and hospitalization.

Result(s)* 142 patients met the inclusion criteria. Patients underwent either primary debulking surgery (PDS) or interval debulking surgery (IDS). 84 patients were treated with conventional management and 58 with ERAS protocol. The mean age for the ERAS group was $60 \pm 13$ vs. $61 \pm 13$ years old for the CM group $(p=0.8315)$. Furthermore, there was no difference between the type of surgery operation duration between the 2 groups. (CM group: 210min vs. ERAS group: 240min, $\mathrm{p}=0.1497 / \mathrm{CM}$ group: $50 \%$ PDS - 50\% IDS vs. ERAS group: $38 \%$ PDS $-62 \%$ IDS, $\mathrm{p}=0.1554)$. However, the occurrence of ICU admittance (32\% vs. $14 \%, p=0.01263)$, overall postoperative complications (32 vs. 22.6, $\mathrm{p}=0.004)$ and hospitalization ( 9 vs. 7 days, $\mathrm{p}<0.001$ ) were significantly reduced by the implementation of the ERAS protocol. Last but not least, concerning 30day mortality: 3 patients died during conventional management, while only 1 died during ERAS protocol.

Conclusion* The implementation of the ERAS program in the management of AOC improves patient's postoperative morbidity, reducing the interval time between surgery and systematic therapy. Less need for the ICU and fewer days in the hospital can decrease healthcare costs in high-volume gynecological oncological centers.

\section{MAINTENANCE OLAPARIB IN PLATINUM-SENSITIVE RECURRENT OVARIAN CANCER: A COMPREHENSIVE CANCER CENTRE'S EXPERIENCE}

I Moreira*, M Ferreira, AR Lopes, J Savva-Bordalo, M Abreu, S Sousa, D Pereira. Portuguese Institute of Oncology of Porto, Medical Oncology

\subsection{6/jgc-2021-ESGO.414}

Introduction/Background* The majority of newly diagnosed patients with ovarian cancer respond to platinum-based chemotherapy (ChT). However, most patients eventually relapse and will need subsequent treatment. Olaparib is a poly ADPribose polymerase inhibitor that has shown efficacy as maintenance treatment in patients with platinum-sensitive relapsed ovarian cancer.

Methodology We retrospectively evaluated patients with platinum-sensitive relapsed ovarian cancer treated with maintenance olaparib (400mg bid, capsules or $300 \mathrm{mg}$ bid, tablets), who previously received $\geq 2$ platinum-based ChT regimens and had a partial or complete response to last platinum-based regimen. All patients were BRCA 1/2 mutated (germline and/or somatic). Study endpoints were progression-free survival (PFS), overall survival (OS), overall response rate and adverse events. Result(s)* Between May 2016 and December 2020, 21 patients were treated with olaparib. Median age was 55 years (range 44-69), and all had ECOG $\leq 1$. The majority had an ovary primary tumour location $(81.0 \%)$ and serous histology (85.7\%). Thirteen patients $(61.9 \%)$ had partial response to most recent platinum-based ChT, and eight (38.1\%) had complete response. Median follow-up time was 18.3 months (1.860.3), with 13 patients alive. Median PFS was 8.3 months (CI95\% 6.0-10.6). Median OS was not reached. Overall response rate was $19.0 \%$ (4 complete responses) and 16 patients had stable disease; hence, 95.2\% benefited from treatment with olaparib. There were no differences in PFS by number of prior platinum regimens, response to last platinumbased ChT, time-to-progression after penultimate platinumbased ChT (>6-12 vs $>12$ months) or BRCA mutation type (germline vs somatic). Most adverse events reported were grade 1 or 2 and were mainly nausea and haemathologic toxicity. Grade 3 and 4 adverse events occurred in six (28.6\%) patients and were: anaemia, neutropaenia and nausea. Thirteen (61.9\%) patients suspended olaparib, 12 (57.1\%) due to disease progression and one due to her own will. There were no patients that suspended treatment due to toxicity.

Conclusion* Our results confirm the effectiveness and safety of maintenance olaparib in real-world setting. This treatment is feasible in the clinic and well tolerated, with manageable toxicity.

\section{METASTATIC HIGH GRADE SEROUS OVARIAN CANCER HAS AN IMMUNE EXCLUDED TUMOR MICROENVIRONMENT - EXPLAINING FAILURE OF IMMUNOTHERAPY TO DATE}

${ }^{1} \mathrm{~K}$ Glennon* ${ }^{2} \mathrm{~A}$ Treacy, ${ }^{3} \mathrm{~K}$ Slattery, ${ }^{3} \mathrm{~S}$ Cunningham, ${ }^{4} \mathrm{JMC}$ Cormack, ${ }^{4} \mathrm{~A}$ Fabre, ${ }^{5} \mathrm{~W}$ Kolch, ${ }^{3} \mathrm{~L}$ Lynch, ${ }^{1,5}$ D Brennan. ${ }^{1}$ UCD School of Medicine, Mater Misericordiae University Hospital, Department of Gynaecological Oncology, Dublin, Ireland; ${ }^{2}$ Mater Misericordiae University Hospital, Department of Pathology, Dublin, Ireland; ${ }^{3}$ Trinity College Dublin, School of Biochemistry and Immunology, Dublin, Ireland; ${ }^{4}$ Conway Institute, UCD School of Medicine, Department of Pathology, Dublin, Ireland; ${ }^{5}$ UCD School of Medicine, Systems biology Ireland, Dublin, Ireland

10.1136/ijgc-2021-ESG0.415 
Introduction/Background* The tumour microenvironment (TME) in metastatic high grade serous ovarian cancer (HGSOC) is not well described. We present a multimodal characterisation of intraepitelial TILs (iTILS) and stromal TILs (sTILS) using flow cytometry (FACS) immunohistochemistry (IHC) in matched primary and metastatic HGSOC samples.

Methodology FACs and IHC for CD4 and CD8 were performed on 26 samples from seven women with HGSOC. Tissue samples, labelled with fluorescent antibodies against CD3, CD4, CD8, checkpoints TIGIT, PD1 and cytokine IFN- $\gamma$ also were analysed with a FACS Fortessa (BD Biosciences). IHC was performed on samples and images annotated to assess intra-epithelial and stromal CD4 and CD8 expression using ImageScope (Aperio), and analysed using the Aperio Nuclear Algorithm v9 (figure 1). Statistical analysis was performed using IBM SPSS 24 or Prism Graph Pad. Quantitative variables were assessed with one way ANOVA and Mann Whitney test.

Result(s)* FACs demonstrated that, compared to primary samples, the frequency of CD8+ TILs $(\mathrm{p}=0.017)$, TIGIT $(p=0.013)$ and PD1 $(p=0.017)$ expression was reduced in matched metastatic sites. CD4+ TILs levels were unchanged between primary and metastatic samples. Consistent with a reduced level of cytotoxic activity, IFN- $\gamma$ on CD8 + TILs was reduced in the metastatic TME $(\mathrm{p}=0.034)$.

IHC demonstrated that the majority of primary samples $(5 /$ 7 (71.4\%) showed a higher proportion of CD8 + sTILs compared to iTILs (figure $3 \mathrm{~b}$ ). In the one BRCA mutated patient, the CD8 iTILs were higher than sTILs (figure 4a). In FACs, this sample also had the highest frequency of CD8+ TILs within the ovarian tumour (figure $4 b$ ).

In IHC from 4/6 different metastatic sites (omentum, vagina, spleen and peritoneum) the density of CD8+ sTILs was higher than iTILs, demonstrating these tumours were immune excluded. Serosal liver and diaphragmatic metastases demonstrated increased CD8+ iTILs compared with primary tumours. Although not as marked, this pattern was replicated in CD4 sTILs (figure 5b, c).

\section{IFN on CD 8 Ovary and Omentum}

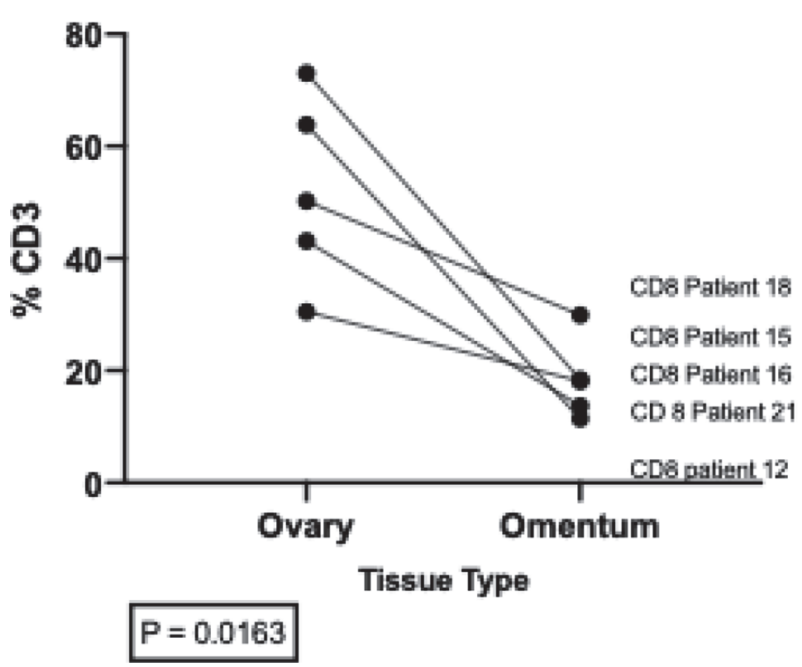

Abstract 498 Figure 2 Flow cytometry IFN- $\gamma$ expression in Primary ovary and metastatic samples

Fig 2a FACs analysis of IFNg expression on CD8+ T cells from primary HGSOC samples in primary ovary, metastatic omental samples. This graph demonstrates that the proportion of IFNg decreases in CD8+ T cells on metastatic $T$ cells when compared to primary ovarian samples

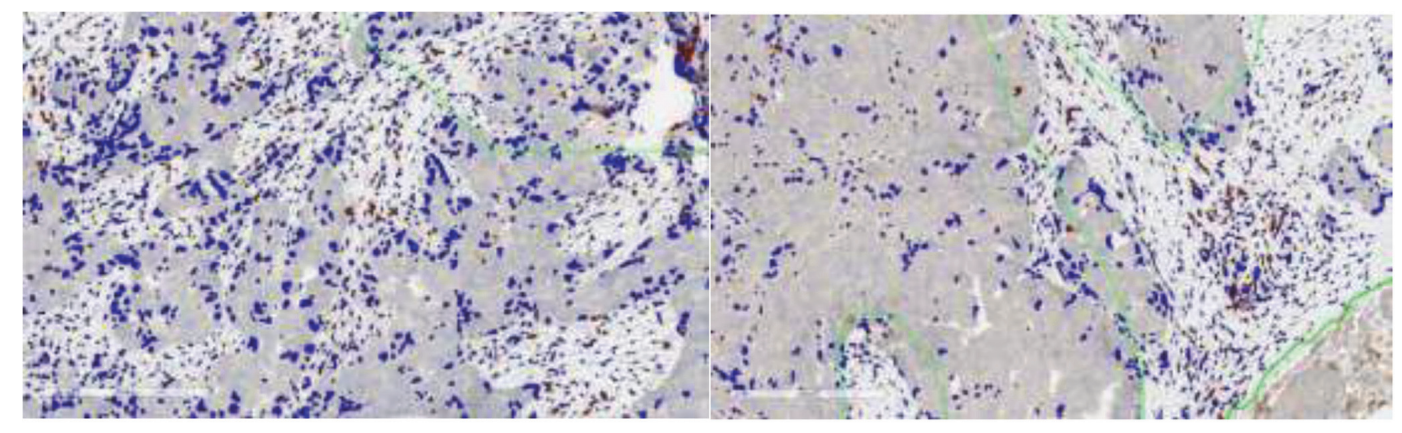

Ovary Sample: CD4 staining

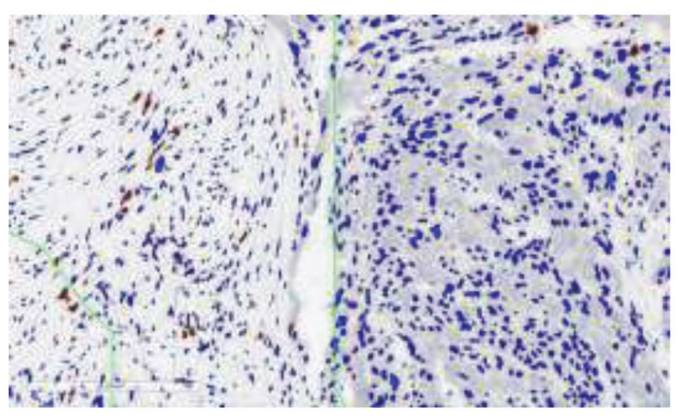

Ovary Sample: CD8 staining

\section{Peritoneum sample - CD4 staining}

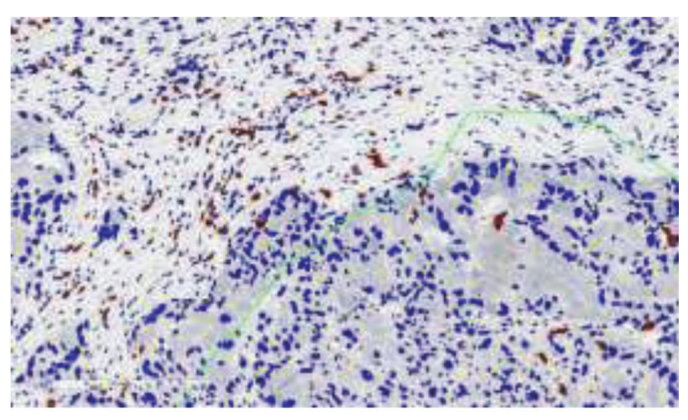

peritoneum CD8 staining

Abstract 498 Figure 1 Immunohistochemistry of primary ovarian HGSOC stained for CD4 and CD8 Demonstration of presence of CD4+ and CD8+ TILS in stroma and ovarian tissue. TIL expression was calculated as the number of cells/mm2 


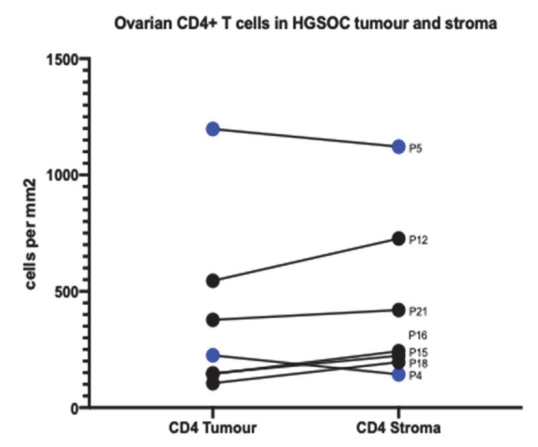

CD4 Location

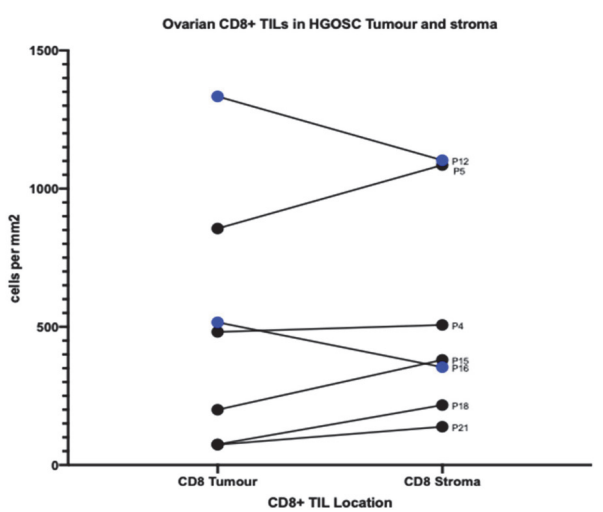

B $p=0.6$

Abstract 498 Figure 3 Immunohistochemistry results of CD4+ T cells and CD8+ TILs in ovarian tumour and stroma $3 a$ This graph shows that in 5/7 (71.4\%) of ovarian tumour samples the number of cells per mm2 of CD4+ T cells was increased in the surrounding stroma compared to the tumour itself. The black dots represent those patients in whom the tumor showed a lower number of CD4+ T cells per mm2; $3 \mathrm{~b}$ This graph shows in $5 / 7(71.4 \%)$ of patients the number of cells per mm2 of CD8+ TILs was greater in the stroma. Therefore these tumours could be considered 'cold' or 'immune excluded'
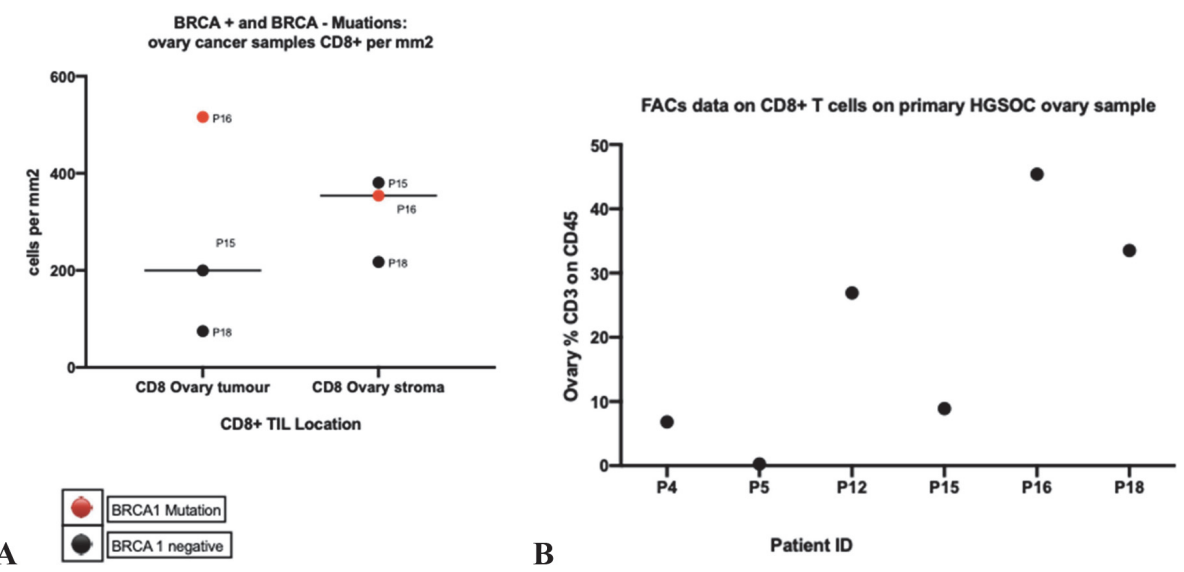

Abstract 498 Figure 4 BRAC status and IHC

4b: Flow cytometry from primary ovarian cancer tissue samples; 4a BRCA mutation was known in three patients. One $(1 / 3,33.3 \%)$ was BRCA1 mutated (p16) and this patient sample showed an increased proportion of CD8+ TILs in the tumour compared to the surrounding stromal tissue 4b Flow cytometry: This demonstrates the levels of CD8+ T cells within the primary ovarian tumour from our patient cohort. The BRCA1 mutation on P16 showed the highest number of CD8+ T cells, correlating with the IHC data.
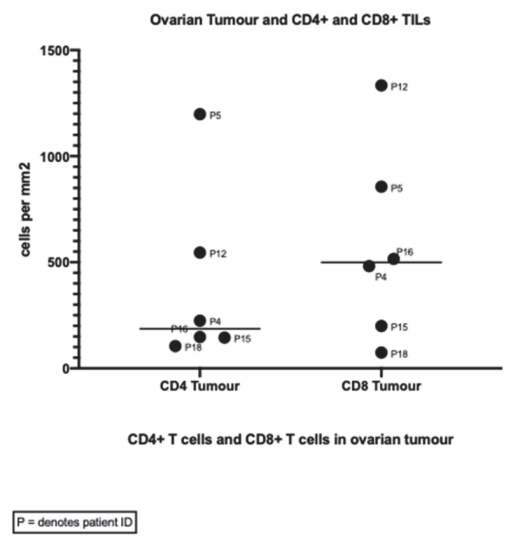

A $p=0.3$

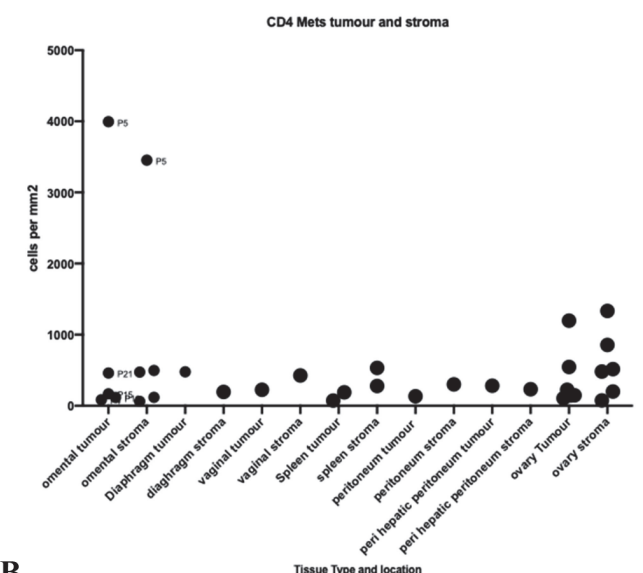

B

Abstract 498 Figure 5 Immunohistochemistry of CD4+ and CD8+ TILs in tumour and stroma HGSOC primary samples

$5 a$ Immunohistochemistry reveals the number of CD4+ and CD8+ T cells per mm2 within the ovarian tumour. A higher proportion of CD8+ TILs was noted within the tumour tissue; Fig $5 b$ A representation of the CD4+ T cells in the HGSOC metastatic samples. For metastatic samples in the omentum $(3 / 5)$ vagina, spleen and peritoneum, the stroma had a greater proportion of CD4+ T cells than the tumour. In the diaphragm and in serosal liver deposits, the tumour was enriched with CD4+ T cells. The difference was less marked than in the CD8+ TILs ( see fig 5c); 5 C A representation of the CD8+ TILs in the HGSOC metastatic samples. For the majority of metastatic samples, the presences of CD8+ TILs in the metastatic cancer samples was lower in the tumour sample than within the stromal tissue 
Conclusion* Using a multimodal approach, including IHC and FACs, we demonstrate that the metastatic TME in HGSOC is significantly different to the primary TME. These findings provide an initial explanation as to why immune checkpoint inhibitors have failed in HGSOC and warrant further investigation.

\section{FERTILITY OUTCOMES FOLLOWING FERTILITY SPARING SURGERY FOR THE MANAGEMENT OF EARLY-STAGE CLEAR CELL OVARIAN CARCINOMA; A SYSTEMATIC REVIEW}

A Prodromidou*, C Theofanakis, N Thomakos, D Haidopoulos, A Rodolakis. st Department of Obstetrics and Gynecology, Medical School, National and Kapodistrian University of Athens, "Alexandra" Hospital, Athens, Greece

\subsection{6/ijgc-2021-ESG0.416}

Introduction/Background* The application of fertility sparing surgery (FSS) in patients with clear cell ovarian carcinoma (CCOC) has been extensively criticized, even in patients with stage IA or IC disease, due to the high reported recurrence rates and the resistance to chemotherapy. The objective of the present study was to evaluate the obstetric and fertility outcomes of patients with early stage CCOC following fertility sparing surgery (FSS).

Methodology Three electronic databases were systematically searched for articles published in the field up to December 2020 using the terms "ovarian cancer", "clear cell", "fertility sparing", "conservative treatment". Studies that reported pregnancy and obstetric outcomes after FSS for the management of early stage CCOC were considered eligible for inclusion.

Result(s)* A total of 5 retrospective studies with 60 patients with CCOC who underwent FSS were included. Mean patients' age was 34.8 years. The total clinical pregnancy rate was $32 \%$ with a proportion of $24 \%$ of live birth rates in 12 of the included patients. The median interval from disease management to pregnancy was 41.5 months. Recurrence rate was $16.6 \%$ among the included patients. Survival and recurrence rates were not different in patients who had FSS compared to those who had radical surgery.

Conclusion* Fertility-sparing treatment for stage IA/IC CCOC seems to be an acceptable treatment option for selected women of reproductive age with a strong desire of fertility preservation. Further larger multicenter studies and studies derived from registries are warranted to validate the special aspects of the procedure and to designate the potential candidates who will receive survival and fertility benefit from fertility-sparing surgery.

\section{SIGNIFICANT SURVIVAL BENEFIT IS ASSOCIATED WITH COMPLETE CYTOREDUCTION IN DELAYED CYTOREDUCTIVE SURGERY FOR ADVANCED OVARIAN CANCER}

V Cassar*, S Rundle, P Korompelis, A Fisher, A Kucukmetin, N Ratnavelu, C Ang. Queen Elizabeth Hospital Gateshead, Gynaecology Oncology, Gateshead, UK

\subsection{6/ijgc-2021-ESG0.417}

Introduction/Background* The current gold standard in the surgical management of advanced ovarian cancer(AOC) recommended by ESGO and ASCO is complete resection of all visible disease. If this is not deemed possible in the upfront setting, then interval cytoreductive surgery should be undertaken after $\leq 4$ cycles of NACT. Occasionally due to the persistence of unresectable sites of disease on interval scanning or because of factors associated with fitness for surgery, surgery in the interval setting may not be possible.

Limited published data assessing outcomes from surgery delayed to after 5 or 6 cycles of NACT(delayed cytoreductive surgery) suggests a potential benefit over no surgery and suggests that if interval cytoreductive surgery is not possible, then the clinician might consider delayed surgery on a case by case basis.

We sought to review the outcomes of patients with AOC presenting to the Northern Gynaecological Oncology Centre (NGOC) who underwent delayed surgery.

Methodology This study is a retrospective analysis looking at patients with AOC referred to the Northern Gynaecological Oncology Centre(NGOC), Gateshead, UK between 2017 and 2020, who were not deemed suitable to undergo either primary cytoreductive or interval debulking surgery and instead had DDS following 5 or 6cycles of NACT .

Result(s)* Over the 3 year period, 401 patients with AOC were referred to the NGOC MDT. 25 patients had delayed surgery, $16(66.7 \%)$ patients had surgery after 5 cycles of chemotherapy, $7(28 \%)$ patients had surgery after 6 cycles and there was incomplete data in 1 patient. The median age was 64. $66.7 \%(16 / 24)$ presented with stage IIIc disease, 12.5\%(3/ 24) were stage IVa and $20.8 \%(5 / 24)$ were stage IVb. The majority had high grade serous carcinoma(91.7\%). All patients had platinum based NACT(Carboplatin), 17 had dual agent chemotherapy with Paclitaxel and 3 had additional Bevacizumab.

18/19 received post-operative chemotherapy and complete cytoreduction was achieved in $17 / 24(71 \%)$ patients. Suboptimal cytoreduction was more common in patients having surgery after 6 cycles and the median overall survival was 24 months in those completely cytoreduced compared to 9.5 months in those with residual disease $(p=0.03)$.

Conclusion* A significant improvement in OS is seen in women who are completely cytoreduced after $\geq 5$ cycles compared to those with residual disease post-operatively. Therefore, if complete clearance can be achieved, cytoreductive surgery should be offered to all patients even after $\geq 5$ cycles.

\section{DIAGNOSTIC FEATURES OF OVARIAN CANCER IN PREMENOPAUSAL WOMEN}

${ }^{1}$ I Pelayo, ${ }^{1} \mathrm{~V}$ Corraliza-Galan*, ${ }^{1} \mathrm{C}$ Martin-Gromaz, ${ }^{1} \mathrm{MJ}$ Pablos-Antona, ${ }^{1} \mathrm{C}$ Del Valle-Rubido, ${ }^{1}$ E Cabezas-Lopez, ${ }^{1} \mathrm{D}$ Rubio-Marin, ${ }^{2} \mathrm{~B}$ Perez-Mies, ${ }^{1} \mathrm{~L}$ Abarca-Martinez, ${ }^{1} \mathrm{E}$ MoratallaBartolome, ${ }^{1} \mathrm{C}$ Sanchez-Martinez, 'I Lazaro de la Fuente. 'Ramon y Cajal Hospital, Obstetrics and Gynecology, Madrid, Spain; ${ }^{2}$ Ramon y Cajal Hospital , Pathology, Madrid, Spain

\subsection{6/ijgc-2021-ESG0.418}

Introduction/Background* Ovarian cancer (OC) is the most lethal gynaecological malignancy worldwide, specially because it's diagnosed as advanced-stage disease. Clinical aspects are inespecific and appear in advanced stages. Ultrasound study (US) remains the primary modality for assessment of ovarian tumors. Computed tomography (CT) imaging is the standard of care for pre-operative evaluation of ovarian cancer patients. Serum CA125 assay has low sensitivity in early stages. 